\title{
Bezold-Jarisch reflex caused by postural change
}

\author{
Yun Hee Kim · Dong Jun Kim • Woon Young Kim
}

Received: 29 August 2013/Accepted: 17 June 2014/Published online: 18 July 2014

(C) Japanese Society of Anesthesiologists 2014

Keywords Bezold-Jarisch reflex $\cdot$ Cushing's syndrome

To the Editor:

The Bezold-Jarisch reflex (BJR) is thought to be peripheral venous blood pooling and a heightened contractile state which result in reflex arterial vasodilation and subsequent vagally mediated bradycardia. The clinical occurrence of BJR in patients with Cushing's syndrome is rare due to its sodium retention and volume expansion. To our knowledge, this is the first report of BJR in a patient with Cushing's syndrome.

A 57 -year-old female (weight $81.65 \mathrm{~kg}$; height $157 \mathrm{~cm}$ ) was scheduled for left adrenalectomy due to Cushing's syndrome. After general anesthesia, postural change from supine to right kidney rest lateral decubitus position elicited a sudden severe hypotension and bradycardia (45-50 mmHg and 35-36 bpm) (Supplementary Fig. 1). Administrated atropine was ineffective. We immediately put the patient in the supine position and her vital signs returned to normal. During that time, all laboratory tests showed normal levels. Electrocardiogram (Supplementary Fig. 2) and transthoracic echocardiogram did not show ischemic signs.

In this case, the impact of body position and the applied weight force on the inferior vena cava (IVC) can have an effect on the central venous return and may provoke BJR. IVC runs along the right side of the aorta and is located slightly to the right of lumbar vertebral bodies (Supplementary Fig. 3A, B). Therefore, IVC is more compressed in the right lateral position than in the supine position. Furthermore, the patient had significant central obesity (her waist was about $96 \mathrm{~cm}$ ) which can cause more significant IVC compression than in a non-obese patient.

Ephedrine is a suitable treatment for BJR and epinephrine must be used early in cardiac arrest. D'Alessio et al. [1] recommended an anticholinergic and $\beta$-blockers against BJR for prophylactic administration of adequate intravenous fluids.

In conclusion, BJR should be considered during right lateral postural change, even if the patient has Cushing's syndrome.

Conflict of interest None.

\section{Reference}

1. D'Alessio JG, Weller RS, Rosenblum M. Activation of the Bezold-Jarisch reflex in the sitting position for shoulder arthroscopy using interscalene block. Anesth Analg. 1995;80:1158-62.
Electronic supplementary material The online version of this article (doi:10.1007/s00540-014-1880-0) contains supplementary material, which is available to authorized users.

Y. H. Kim · D. J. Kim · W. Y. Kim (ه)

Department of Anaesthesiology and Pain Medicine, Ansan

Hospital, Korea University College of Medicine, Gojan 1-dong,

Danwon-gu, Ansan, Republic of Korea

e-mail: ckssis@korea.ac.kr 